Cahiers d'études italiennes

\title{
Banditi e pirati nella narrativa medievale: alcuni casi di fuorilegge cortesi
}

Federica Veglia

\section{OpenEdition}

\section{Journals}

Edizione digitale

URL: http://journals.openedition.org/cei/850

DOI: $10.4000 /$ cei.850

ISSN: 2260-779X

\section{Editore}

UGA Éditions/Université Grenoble Alpes

\section{Edizione cartacea}

Data di pubblicazione: 15 mai 2007

Paginazione: 9-29

ISBN: 978-2-84310-096-3

ISSN: 1770-9571

Notizia bibliografica digitale

Federica Veglia, " Banditi e pirati nella narrativa medievale: alcuni casi di fuorilegge cortesi », Cahiers d'études italiennes [Online], 6 | 2007, Messo online il 15 novembre 2008, consultato il 10 décembre 2020. URL : http://journals.openedition.org/cei/850 ; DOI : https://doi.org/10.4000/cei.850 


\title{
BANDITI E PIRATI NELLA NARRATIVA MEDIEVALE
}

\author{
ALCUNI CASI DI FUORILEGGE CORTESI
}

\author{
Federica Veglia \\ Biella
}

Di predoni di strada e pirati dei mari la letteratura medievale è estremamente ricca. Si tratta di uomini dai profili negativi, che spesso anche la fortuna, dopo averli temporaneamente assecondati, colpisce duramente, attraverso le mani di una legittima giustizia, terrena o divina che sia. C’è una figura, però, che emerge dal gruppo di questi personaggi abietti e suscita un particolare interesse per il lettore: si tratta del fuorilegge che adotta come principi etici assoluti la liberalità e la magnanimità, e che, pur compiendo azioni ritenute colpevoli dall'autorità legittima, è considerato in qualche modo eroe positivo dalla comunità a cui appartiene. Con un'espressione sintetica, anche se approssimativa, potremmo definire 'fuorilegge cortese' tale figura letteraria.

Essa è presente in maniera significativa, anche se quantitativamente circoscritta, nella produzione letteraria italiana del Trecento e Quattrocento, e si manifesta in forme letterarie della tradizione alta e della tradizione popolare anche in altri Paesi europei (si pensi al Robin Hood della tradizione inglese); attraversa poi l'età moderna e riemerge con insistenza alla fine del Settecento e lungo tutto l'Ottocento, dando vita a numerosi personaggi di questo tipo, attivi in diversi codici artistici (dalla narrativa al teatro, all'opera lirica, più avanti al cinema) ${ }^{1}$. In essa, elementi

1. Riguardo al personaggio di Robin Hood, il testo di riferimento per la tradizione medievale è R.B. DoBSON-J. TAYLOR, Rhymes of Robin Hood, London, Heinemann, 1976. I più antichi testi rimasti che hanno per protagonista il personaggio sono ballate, probabilmente composte nel XIV secolo, ma i manoscritti più antichi risalgono al 1450 e non è possibile stabilire una datazione certa. Del resto, gli storici stessi non sono concordi nemmeno sulla ricostruzione del personaggio storico di Robin conte di Huntington (il probabile referente storico per la costruzione della leggenda) e alcuni negano che vi sia un'unica figura storica di riferimento. Quanto a personaggi di questo tipo tra Settecento e Ottocento, essi sono numerosissimi in Europa: basti citare testi come I masnadieri [Die Räuber] di Schiller (1781, da cui è tratta l'omonima opera musicata da Verdi) e Il corsaro di Byron (1814), da cui l'opera di Bellini Il pirata (1827) e di Verdi Il corsaro (1848). 
storici e trasfigurazione leggendaria e letteraria si intrecciano in una maniera del tutto peculiare, che merita un'attenzione specifica da parte dello storico della letteratura.

Mia intenzione è proprio di verificare la presenza e la portata di tali personaggi nella tradizione letteraria italiana del Tre-Quattrocento (in particolare nella novellistica), anche in rapporto con le figure di altri banditi non cortesi; di individuarne eventuali fonti; di scoprire resistenze oppure filoni particolarmente fecondi; di interpretare le testimonianze letterarie nel loro stretto legame con gli eventi e i personaggi storici a cui si riferiscono.

Il fenomeno dell'insorgenza di tali figure leggendarie e letterarie è stato in parte analizzato da Eric Hobsbawm a proposito di quello che egli chiama "banditismo sociale», di cui una specifica forma si incarna nella figura del «bandito-gentiluomo», secondo la sua stessa definizione, ossia del bandito di nobile origine che ha comportamenti liberali. Con l'espressione di «banditismo sociale» Hobsbawm si riferisce all'emergenza, in aree contadine in genere marginali, soggette ad un dominio non accettato e in momenti di forte cambiamento sociale, di persone o gruppi di persone che commettono azioni considerate punibili dalla legge e dall'autorità costituita ma non considerate colpevoli dalle comunità di appartenenza. Il fatto storico che secondo lo studioso suscita il maggiore interesse, però, è proprio la trasfigurazione leggendaria che di tali personaggi viene data: secondo l'autore essa è originata dalla necessità di idealizzare, e rendere compatibile con le aspirazioni di cambiamento e rinnovamento, un comportamento che si dimostra innanzi tutto libero e privo di legami, noncurante dell'autorità, ispirato a valori di equità assoluta e moderazione nell'uso della violenza, e spesso determinato da un torto iniziale subito ingiustamente ${ }^{2}$. Ebbene, a quanto consta dalle ricerche effettuate dallo studioso in diversi contesti geografici e storici, anche se non nel Medioevo italiano, la trasfigurazione leggendaria e letteraria delle figure di fuorilegge di nobile origine che diventano paladini dei deboli e raddrizzatori dei torti

2. Riguardo ai «banditi sociali», Hobsbawm traccia il ritratto di persone «ritenute criminali dal signore e dall'autorità statale, ma che pure restano all'interno della società contadina e sono considerate dalla loro gente eroi, campioni, vendicatori, combattenti per la giustizia, persino capi di movimenti di liberazione e comunque degni di ammirazione, aiuto, appoggio» (Eric HoBSBAWM, I banditi. Il banditismo sociale nell'età moderna, Torino, Einaudi, 1971, pp. 11-12). Per quanto riguarda invece la categoria specifica del «bandito-gentiluomo», tra le caratteristiche che Hobsbawm individua attraverso un'analisi contrastiva dei testi e delle testimonianze vi è per esempio il fatto che egli «non uccide, se non per autodifesa o per giusta vendetta», che «inizia come vittima di un'ingiustizia o perseguitato per un'azione che l'autorità, ma non la sua gente, giudica criminosa», che "prende dal ricco per dare al povero" (ibidem, p. 37). 
ha un successo straordinariamente più ampio e duraturo di quella del bandito di provenienza contadina ${ }^{3}$.

Perché, però, e come proprio alcune figure e non altre subiscano questa sorta di trasfigurazione è una domanda a cui è molto difficile rispondere; nel campo d'indagine che ho scelto di considerare, per esempio, risulta difficile comprendere come mai di un personaggio come Ghino di Tacco nasca e si sviluppi una fama leggendaria che collima con tutte le caratteristiche enunciate da Hobsbawm, senza che alcun documento storico ci lasci testimonianza di un suo agire particolarmente magnanimo o liberale, mentre di altri banditi l'immagine letteraria sia totalmente ribaltata. Sarebbe interessante scoprire davvero il percorso storico attraversato da tali nodi leggendari e potere così dare una spiegazione delle straordinarie affinità tra personaggi come Ghino e Robin Hood. Per far questo, penso che sia necessaria innanzitutto una buona documentazione storica e storico-letteraria, e accanto un'analisi mirata delle risultanze letterarie di tale processo di costruzione leggendaria.

Ghino di Tacco è il primo e, per quantità e qualità d'attestazioni, più significativo personaggio che la tradizione letteraria italiana ci presenta con i tratti del bandito-cortese; l'unico, per ora, per il quale sia possibile confrontare ampiamente documenti storici e testimonianze letterarie ${ }^{4}$. Uomo della nobiltà feudale della Val di Chiana, di famiglia ghibellina, risulta pluri-condannato a morte dal comune di Siena, e di lui si hanno notizie nei documenti degli Archivi senesi tra il 1285 e il 1296. Si deve ad un avvincente articolo di Giovanni Cecchini una ricostruzione dettagliata e rigorosa di ciò che si può ancora sapere della vita del personaggio, corredata dalla pubblicazione in appendice di tutti i documenti ritrovati che lo riguardano ${ }^{5}$. In sintesi: la famiglia della Fratta, della quale fa parte Ghino,

3. Ibidem, p. 36. Riguardo al «bandito-gentiluomo» (o «ladro-gentiluomo»), Hobsbawm sostiene che «il suo ruolo è quello del campione, del vendicatore dei torti, del difensore della giustizia e dell'equità sociale. Le sue relazioni con i contadini sono di solidarietà e identità totali» (ibidem, p. 37).

4. Sull'argomento si vedano Ghino di Tacco nella tradizione letteraria del Medioevo, a cura di Bruno Bentivogli, Roma, Salerno Editrice, 1992; ID., La vendetta di Ghino di Tacco. Per il commento a Purgatorio VI, 13-14, in "Filologia e critica», XVI (1991), pp. 267-271; Franco CardINI, Ghino di Tacco: una storia toscana fra medioevo mitografico e Medievistica, in La Val d'Orcia nel medioevo e nei primi secoli dell'età moderna, Atti del Convegno internazionale di studi storici (Pienza, 15-18 settembre 1988), a cura di A. Cortonesi, Roma, 1990.

5. Giovanni CeCCHInI, Ghino di Tacco, in «Archivio storico italiano», CXV (1957), pp. 263298. 
appartenente al ceppo dei Cacciaconti, perde il dominio feudale sulla zona della Fratta e di Torrita già dagli inizi del Duecento, a causa dell'espansione del comune senese; riesce però a conservare i propri diritti e una sostanziale indipendenza ancora per diversi anni, intrattenendo buoni rapporti con tale comune. Sennonché l'avvento della parte guelfa rende sempre più difficili i rapporti con le famiglie feudali ghibelline e $\mathrm{i}$ fuoriusciti ghibellini da Siena si uniscono ad alcuni nobili ghibellini della zona per azioni di disturbo contro il comune; tra costoro, sono attivi il padre di Ghino, Tacco, e lo zio, Ghino. Contro di loro sono emesse diverse condanne, che si fanno sempre più gravi a mano a mano che la situazione diventa più difficile per la città, sino al bando assoluto dall'intero contado.

Tacco e il fratello, insieme agli altri, si danno alla macchia, ottenendo grande appoggio, a quanto consta, in diversi luoghi della zona, e possono vivere impunemente per parecchio tempo. Interessante, per esempio, un documento in cui viene emanata una condanna contro il nipote dei due fratelli (Ghino, figlio di Federico, il quale aveva raggiunto gli zii nella macchia), con l'accusa di avere rubato diversi maiali nella zona (nella silva communis Torrita). Questa è la vita quotidiana dei fuorusciti, che devono senz'altro godere dell'appoggio di un buon numero di persone per non essere scoperti, ma che conducono pur sempre una vita irregolare, per affrontare la quale è necessario essere dotati di grandi capacità di adattamento. Soprattutto, è una vita che si svolge interamente al di fuori degli spazi sociali abitati o in qualche modo antropizzati. Coraggio, adattamento ad una natura che per la comunità umana è ostile (la montagna, non a caso) e alterità spaziale: forse queste, a ben vedere, sono tra le caratteristiche che favoriscono la loro trasformazione letteraria in figure eccezionali.

Alla vita da banditi, poi, probabilmente partecipa fin da molto presto anche Ghino, figlio di Tacco, la cui madre, forse della famiglia dei Tolomei, trova asilo, dopo la condanna del marito, in un palazzo senese. Finché il giudice vicario del podestà di Siena, l'aretino Benincasa da Laterina (che sarà citato da Dante) non riesce a fare arrestare Tacco e a farlo giustiziare (1285). A partire dal 1288, tre anni dopo, sono attestate condanne anche nei confronti del figlio Ghino, «qui fecit insultum in stratam publicam». La pena a cui è condannato, come fa notare Cecchini, non è quella prevista per un reato privato come il latrocinio di strada, ma è una pena equivalente a quella per un reato contro il comune stesso. Anche Ghino, dunque, viene considerato nemico "pubblico» (più avanti 
verrà definito «rebelle et inimico manifesto communis et populi senensis»).

Altri documenti per noi interessanti sono quelli che attestano, tra il 1294 e il 1296, il tentativo di alcuni nobili locali di aiutare Ghino ad insediarsi nel castello delle Serre, e infine il suo sicuro insediamento nel castello di Radicofani, in un territorio nominalmente della Chiesa, ma su cui accampa diritti il comune di Siena (nella realtà, quindi, territorio di nessuno, in cui facilmente Ghino riesce a trovare rifugio stabile). L'appoggio nei confronti del fuoruscito, a questo punto, rivela nettamente un'origine più propriamente politica che sociale, legata cioè ad aspirazioni di potere più che di riscatto sociale, il che sarà del tutto assente dai documenti letterari che considereremo. Da qui in poi le notizie dei documenti d'archivio si interrompono. Ė probabilmente vera la notizia che dà Dante della vendetta di Ghino sul giudice Benincasa (non si vede perché, a questo proposito, Dante avrebbe dovuto intenzionalmente dare una notizia falsa), ma le fonti che abbiamo non lo accertano. Così come assolutamente nulla ci dicono di eventuali azioni liberali o magnanime nei confronti di chicchessia.

E qui che interviene la "trasfigurazione", o meglio, seguendo le teorie di Hobsbawm, l'applicazione al personaggio, che doveva avere in qualche modo fascinato i contemporanei, dei caratteri peculiari che il cosiddetto bandito ideale ha per una comunità che mal sopporta un'autorità estranea. Di tale trasfigurazione non possediamo altro se non testi letterari (o di commento letterario) e dunque ci è difficile ricostruire, se davvero è esistita, una leggenda popolare sul personaggio; cioè, sempre che Hobsbawm abbia ragione, ricostruire quanto di quello che i nostri autori dicono appartenga ad una tradizione popolare che vede nel ribelle una possibilità di riscatto, quanto alla tradizione letteraria alta (in particolare alcuni testi antichi), quanto ad invenzioni o reinvenzioni dei singoli autori.

L'excursus tra le testimonianze letterarie che nominano Ghino di Tacco non seguirà un ordine cronologico, perché preferisco prendere avvio dal testo che per primo fissa nel personaggio in maniera compiuta alcuni dei caratteri del bandito-gentiluomo, ovvero la novella seconda della X giornata del Decameron. Considererò in seguito due testi dei primi decenni del Trecento, ossia i Documenti d'Amore di Francesco da Barberino e la Commedia che, pur senza attribuire esplicitamente al personaggio i caratteri del bandito gentiluomo, si riferiscono implicitamente ad una sua fama eccezionale, perlomeno ambivalente; infine, prenderò in esame alcuni commenti antichi alla Commedia, influenzati o meno dal testo boccacciano, primo su tutti il commento di Benvenuto da Imola. 
Dunque, la novella di Boccaccio. A ben vedere, qui il protagonista di azioni «magnifiche», secondo il volere della narratrice Elissa, è l'abate di Cluny, non il «rubatore delle strade» Ghino di Tacco ${ }^{6}$. Addirittura c'è chi sostiene, come Santini, che il comportamento di Ghino non sia per nulla ispirato a cortesia e liberalità, e che anzi la sua azione sia tutta tesa a sminuire la dignità dell'abate e a privarlo della sua posizione dominante più che a dimostrargli magnificenza e liberalità ${ }^{7}$. Non credo che le cose stiano così: mi sembra che il personaggio che campeggia, in tutta la narrazione, sia proprio Ghino, la cui effettiva comparsa nella storia viene ritardata, con studiata tensione narrativa, fino all'incontro finale con l'abate e alla proposta indiscutibilmente magnanima (il consentirgli di riprendersi l'intero bottino di cui era stato privato). L'abate ottiene poi per lui una prioria nell'Ordine degli Ospitalieri, ma ciò non mi pare sminuisca la posizione centrale del bandito e il rilievo dato alla sua azione (il personaggio risulta onnipresente, direi, nella novella): l'epilogo della storia, in effetti, risulta più una chiosa aggiuntiva che non il momento culminante $\mathrm{e}$ risolutivo della vicenda. Tale momento culminante si conclude e si risolve con la risposta che l'abate dà alle proposte del brigante ( MMaravigliossi l'abate che in un rubator di strada fosser parole sì libere [...]. "Maledetta sia la fortuna, la quale a sì dannevole mestier ti costringe!”»; (X, 2, 24). Risposta che fornisce una completa riabilitazione morale del personaggio, deresponsabilizzandolo per quei comportamenti su cui il narratore aveva in precedenza sospeso il giudizio.

Al di là del ruolo di protagonista attribuibile o meno al personaggio, penso che più interessante sia concentrarsi sui modi che Boccaccio sceglie per la rappresentazione di una figura potenzialmente così ambigua. La valutazione della vicenda e dei due personaggi si gioca tutta sul dialogo finale tra i due protagonisti, mentre la presentazione iniziale e i comportamenti durante lo svolgimento dell'azione non sono accompagnati da alcun giudizio univoco. Il giudizio su di essi rimane sospeso, e sul personaggio di Ghino in particolare in tutta la novella, sino al dialogo risolutivo, prevale un'impressione di mistero non risolto, più che un positivo riconoscimento di eroismo. Il ritratto iniziale lo presenta come uomo famoso contemporaneamente per la sua «fierezza» e per le sue «ruberie»,

6. "Che direm noi se si racconterà un chierico aver mirabil magnificenzia usata verso persona che, se inimicato l'avesse, non ne sarebbe stato biasimato da persona? [...] La qual cosa, cioè come un chierico magnifico fosse, nella mia seguente novella potrete conoscere aperto" (Giovanni BocCaccio, Decameron, a cura di Vittore Branca, Milano, Mondadori, 1992, p. 810).

7. Emilio Santini, La novella di Ghino di Tacco e dell'abate di Clignì, in "Lo spettatore italiano», VII (1954), pp. 491-495. 
quasi il narratore volesse lasciare intravedere il fascino che il personaggio esercita con la sua condotta di vita, ma allo stesso tempo intendesse mantenere una posizione ambivalente e prudente nei suoi confronti, senza apertamente cedere:

Ghino di Tacco, per la sua fierezza e per le sue ruberie uomo assai famoso, essendo di Siena cacciato e nimico de' conti di Santafiore, ribellò Radicofani alla Chiesa di Roma: e in quel dimorando, chiunque per le circustanti parti passava rubar faceva a' suoi masnadieri. $(\mathrm{X}, 2,5)$

Lo stesso termine "fierezza», come vedremo a proposito del passo dantesco in cui è nominato Ghino, con il quale senz'altro il testo di Boccaccio è connesso, ha significato duplice: come per l'eroe pagano Fierabras, fierezza vale allo stesso tempo nobiltà e ferocia. In ogni caso, una dote d'eccezione. Nel corso della novella, poi, soprattutto grazie all'intervento degli ambasciatori, al silenzioso accerchiamento operato dai masnadieri, ai tratti del luogo e di tutto l'ambiente che lo circonda, la sua eccezionalità, già intravista in quella connotazione iniziale, arriva a risultare predominante. Così il personaggio acquista autorevolezza e fascino agli occhi del narratore e del lettore, e grazie a questa autorevolezza conquistata il suo gesto magnanimo risulterà anch'esso eccezionale.

Altri aspetti da considerare, l'origine nobiliare del personaggio e gli eventi che lo hanno portato a diventare «rubatore delle strade». E Ghino stesso ad affermarli nel suo discorso all'abate:

Menò allora Ghino l'abate nella sala dove erano i suoi arnesi e la sua famiglia tutta, e fattolo ad una finestra accostare donde egli poteva tutti i suoi cavalli vedere, disse: «Messere l'abate, voi dovete sapere che l'esser gentile uomo e cacciato di casa sua e povero e avere molti e possenti nimici hanno, per potere la sua vita difendere e la sua nobiltà, e non malvagità d'animo, condotto Ghino di Tacco, il quale io sono, a essere rubatore delle strade e nimico della corte di Roma [...]» (X, 2, 20-21)

Gli elementi che delineano il Ghino di Boccaccio come un fuorilegge cortese, dunque, sono diversi: la pratica di un comportamento liberale e magnanimo, l'effettiva origine nobile del personaggio e la sua caduta a causa di un torto subito, la sua scelta eroica di vivere alla macchia senza compromessi. Ebbene, è possibile ricostruire delle fonti letterarie per questi tratti?

Per quanto riguarda il torto subito da un personaggio nobile e la sua conseguente scelta di uscire dalla società civile e di vivere alle sue spese, ho riscontrato perlomeno un'attestazione antica, nella tradizione romanzesca. Nelle Etiopiche di Eliodoro, Tiamis, il capo dei pirati che rapiscono i due giovani innamorati appena sbarcati in Egitto, a loro volta già vittime di un altro rapimento fallito, è il figlio di un sacerdote egiziano, che è stato 
defraudato dal fratello della legittima successione al padre. Si dà dunque alla macchia e diventa capo di uno dei gruppi pirateschi della zona del Delta del Nilo, dove, secondo la rappresentazione di Eliodoro, un'intera comunità di persone vive dei proventi della pirateria. La sua figura straordinaria risalta immediatamente, le sue qualità di capo sono quelle proprie di un buon comandante militare, e, soprattutto, il suo comportamento si dimostra del tutto liberale per esempio con l'anteposizione della straordinaria bellezza della fanciulla all'intero bottino ${ }^{8}$. Lo scioglimento della trama porterà naturalmente ad un riequilibrio del torto subito, e il nobile Tiamis potrà felicemente ricongiungersi con il padre e rioccupare la posizione sociale che gli spetta.

Senz'altro un prototipo del bandito gentiluomo, anche se il motivo viene sviluppato da Eliodoro soltanto nei tratti funzionali allo sviluppo della vicenda. Del suo rapporto con i più deboli, come del resto avviene anche nella novella di Boccaccio, si intravede soltanto una breve esemplificazione, attraverso l'incontro che lo lega al personaggio protagonista (nel caso di Ghino si tratta del co-protagonista), e attraverso l'autorevolezza conquistata tra i suoi. Probabilmente, in entrambi i casi il personaggio è già presente come un "tipo" nella mente e nella cultura degli autori, e sfugge dunque a ulteriori caratterizzazioni individuali.

Vediamo allora quali tracce ci restano, per il personaggio di Boccaccio, della tradizione che si è creata intorno al personaggio.

Nella Commedia, nel VI canto del Purgatorio, viene nominato un episodio della vicenda di Ghino: "Quiv'er l'Aretin che da le braccia / Fiere di Ghin di Tacco ebbe la morte» (vv. 13-14). Come ho già scritto qualche anno fa, penso che Dante, evocando Ghino nel Purgatorio come assassino di Benincasa e non nominandolo affatto nel girone dell'Inferno in cui sono puniti "guastatori e predoni» (Inf. XII), dimostri di tenere in considerazione in qualche modo una fama quantomeno ambigua del personaggio, e in particolare intenda creare con essa una sorta di compromesso'. Il nesso «braccia fiere», peraltro in forte enjambement, di per sé ambivalente e già presente nella tradizione della chanson de geste, consente a Dante di non prendere partito per nessuna delle due interpretazioni e di non offendere, quindi, una tradizione di Ghino di Tacco che probabilmente è

8. Eliodoro, Le Etiopiche, a cura di A. Colonna, Torino, UTET, 1987, I, 1-4.

9. Cfr. Federica Veglia, Predoni e banditi nella Commedia (a proposito del 'bandito-cortese'), in Leggere Dante, a cura di Lucia Battaglia Ricci, Ravenna, Longo, 2002, pp. 307-321. 
ormai leggendaria.

Credo che se avesse inteso condannare esplicitamente la sua ferocia, e avesse quindi collocato il personaggio insieme agli altri predoni che "fecero alle strade tanta guerra", in Inf. XII, Dante avrebbe fornito in questo modo una condanna esemplare di tutta la fama costruitasi intorno al personaggio. Proprio il fatto che ciò non sia avvenuto, e che il personaggio goda di un privilegio indiscutibile, testimonia innanzitutto che tale fama è già attiva, e allo stesso tempo che Dante non intende opporlesi. È invece del tutto improbabile che sia Dante stesso il primo a voler suggerire un'ambivalenza nella valutazione del personaggio ed eventualmente a dare il via ad una sua trasfigurazione leggendaria.

Che il passo sia da considerare in stretta relazione e richiamo con quello di Inf.XII, poi, è suggerito non solo dall'affinità tematica, ma anche dal fatto che alcuni dei personaggi là elencati, in particolare Rinieri da Corneto e Rinieri dei Pazzi, hanno non poco in comune con Ghino ${ }^{10}$. Di Rinieri dei Pazzi sappiamo che appartenne ad una nobile famiglia ghibellina che esercitava diritti feudali sul contado di Firenze, che fu poi bandito dal comune, e fu artefice di un'imboscata ai danni di un vescovo di passaggio verso Roma e del suo conseguente omicidio ${ }^{11}$. Le analogie con la figura di Ghino di Tacco, in particolare con ciò che di lui rappresenterà Boccaccio, sono notevoli: la differenza, in Boccaccio, è l'esito esattamente ribaltato delle vicende; in Dante, la caratterizzazione di fierezza e un'evocazione nel solo Purgatorio, che lascia perlomeno un dubbio sul suo effettivo destino ultraterreno. Ciò che ha spinto Dante verso questa scelta è probabilmente stata la fama creata intorno all'uomo, più o meno supportata da fatti certi; e si noti che nella novella di Boccaccio, tra i primi aggettivi usati per la connotazione del personaggio, compare l'aggettivo «famoso».

Di Rinieri da Corneto, invece, non abbiamo alcuna testimonianza documentaria. Qualcuno, però, come Michele Barbi, lo identifica con quel Rinieri presente proprio in un altro dei testi di inizio Trecento in cui si parla di Ghino di Tacco, i Documenti d'Amore di Francesco da Barberino ${ }^{12}$. In quel passo, egli si trova in competizione con Ghino e sperimenta a sue spese la settima delle regole sub industria, di cui la storia dei

10. I due personaggi compaiono nei vv. 135-138: «[...] e in etterno munge / le lagrime, che col bollor disserra, / a Rinier da Corneto, a Rinier Pazzo, / che fecero a le strade tanta guerra» (Inf. XII, 135-138). 31.

11. Cfr. Umberto Carpi, I tiranni (a proposito di Inf. XII), in «L'Alighieri», 39 (1998), pp. 7-

12. Michele Barbi, Problemi di critica dantesca, I serie, (1893-1918), Firenze, Sansoni, 1934, 
due personaggi costituisce un exemplum ( Meglio divider che perder la preda / e danno ognun leggero / chi sa portar e saggio / per riparar contra laltro che maggio»" ${ }^{13}$ ). Ecco il brano narrativo che fa da esemplificazione:

Amideus de Brixia mercator ibat Romam cum duobus equis et uno discreto famulo invenit quedam robatorem nomine Raynerium qui austulit ei equos et pannos quo facto supervenit Ghinus de Tacco et dixit Raynerio: "quomodo es tu ausus sine mea licentia hoc fecisse". Dixit ille: "et quomodo vis tu privilegium in rapina qui non proficis ad tuitionem?". Tunc Ghinus obmissis aliis dixit: "aut da michi unum ex istis equis aut auferam a te ambos". Erat enim iste Ghinus potentior valde, ille autem respondit nolens, cum eo fuit in rixa et perdidit ambos quos cum abduceret obviavit mercatori et narravit ei hoc totum. Tunc mercator dixit ad eum verba istius regule ita ex hiis motus Ghinus unum restituit sibi equum. ${ }^{14}$

Rinieri non è in grado di accettare né le norme di comportamento che regolano la vita sociale e consentono l'utile finale di ognuno in qualunque circostanza, anche nel caso di rapine, e neppure la logica del più forte (Ghino è "parecchio più forte» di lui, e per questo è in grado di scegliere come concludere una trattativa). Il suo personaggio è del tutto perdente. Ghino, da parte sua, pur comparendo anch'egli come un usurpatore (come osa il rubator meno forte depredare un viandante senza la sua licentia?), sa rendere attiva in concreto la norma a cui conforma il proprio comportamento anche quando lo svantaggio apparente ricade su di lui (decide dunque di restituire al derubato metà del bottino). Accortezza e piena attuazione della norma enunciata, con una tale capacità etica da non esitare un attimo («ex hiis motus», dice il narratore subito di seguito alle parole del mercante) di fronte ad un gesto che avvantaggia un'altra parte. Il bandito agisce dunque in maniera liberale: non subordina la propria azione al proprio esclusivo vantaggio, ma riconosce una regola morale superiore.

Su Ghino, dunque, paiono essersi addensate qualità morali diverse: magnanimità, capacità oratorie, rigore morale. Sempre più sono convinta che Dante non abbia alcun interesse, per quanto non condivida i metodi violenti di Ghino, ad essere provocatorio o apertamente ostile nei confronti della sua fama.

Ritornando alle tracce di tale fama, ad essa accennano senza metterla in

p. 300. Sul parallelo tra il passo di Francesco da Barberino e i personaggi di Dante, si veda anche Oreste Antognoni, Saggio di studi sulla Divina Commedia, Livorno, Giusti, 1893, pp. 67ss.

13. I documenti d'Amore di Francesco da Barberino secondo i manoscritti originali, a cura di Francesco Egidi, Roma, Società Filologica Romana, 1912, vol. II, p. 96.

14. Ibidem, pp. 96-97. 
alcun modo in discussione tutti i commentatori antichi alla Commedia, che in parte risultano essere l'uno fonte dell'altro, a catena, in parte ricavano le loro notizie direttamente dal racconto boccacciano.

Jacopo della Lana, prima del testo di Boccaccio, commenta così il personaggio dantesco:

[...] Cervo, fratello d'uno Ghino di Tacco, il quale era un grande rubatore e gentile uomo del contado di Siena [...]. Aveva una tale usanza lo detto Ghino, che mai né sofferì, né volle, che persona, ch'elli avesse, morisse in prigione.

È la prima attestazione esplicita di un tratto così evidente del bandito cortese: Ghino non usa la violenza in maniera gratuita, e si prende cura di chi è sotto la sua prigionia, trasformando la prigionia stessa in una sorta di protezione. È senz'altro significativo che l'episodio scelto da Boccaccio, pochi anni dopo, riguardi proprio una prigionia inflitta dal personaggio, ma rivelatasi in ultima analisi una cura benefica. È difficile ricostruire con precisione da quale patrimonio Jacopo della Lana attinga per fornire queste informazioni, certo non dal passo dantesco.

Con gli stessi argomenti e le stesse notizie, poi, chiosano l'Ottimo e Jacopo Alighieri ${ }^{15}$; tornando più fedelmente al testo dantesco, a cui aggiunge però questa ulteriore notizia, Francesco da Buti («Non consentendo mai lo detto Ghino che nessuno, che n'avesse in prigione morisse, con tutto che fosse fiero e violento omo»). Sono, queste, attestazioni che si collocano, a mio parere, a metà tra la storia della leggenda e la costruzione della leggenda stessa.

Il più accanito sostenitore e diffusore della fama di Ghino di Tacco, tra i commentatori della Commedia, è però Benvenuto da Imola, che dedica un'ampia parte del proprio commento ai versi in cui compare il bandito. Benvenuto si riferisce a Boccaccio e all'episodio dell'abate di Cluny, ma aggiunge parecchie altre informazioni, che rivelano in maniera compiuta $\mathrm{i}$ caratteri del bandito cortese:

Ideo, Lector, volo quod scias quod iste Ghinus non fuit ita infamis ut aliqui scribunt, quod fuerit magnus sicarius, et spoliator stratarum. Iste namque fuit vir mirabilis, membratus, niger pilo, et carne fortissimus, ut Scaeva levissimus, ut Papirius Cursor prudens et largus. Fuit de nobilibus de la Fratta, comitatus Senarum. Qui expulsus viribus comitum de Sancta Flora occupavit castrum nobile Radicofani contra Papam. Et cum suis famulis manipulariis faciebat multas et magnas praedas, ita quod nullus poterat ire tutus Romam vel alio per partes illas. Sed fere nullus incurrebat in manus

15. «Guido di Tacco, il quale era un grande rubatore [...] ed avendo il detto Ghino una tale usanza che mai non sofferìo, né volle, che persona ch'elli miesse [avesse] morisse in prigione» (OTTIMO); «Fuit magnus depredator, tamen nullos captos occidebat» (JACOPO AlighiERI). 
eius, qui non recederet contentus, et amaret et laudaret eum. Et audi morem laudabilem in tali arte latrocinandi: si mercator erat captus, Ghinus explorabat placibiliter quantum ille poterat sibi dare; et si ille dicebat quingentos aureos, auferebat trecentos, et reddebat ducentos, dicens: Volo quod possis negotiari et lucrari. Si erat unus sacerdos dives et pinguis, auferebat sibi mulam pulcram et dabat ei unum tristem roncinum. Et si erat unus scholaris pauper vadens ad studium, donabat sibi aliquam pecuniam, et exhortabatur ipsum ad bene agendum et proficiscendum in scientia [...] De homine isto plura non dico, de quo posset fieri Tragoedia.

Non perché fu rubatore delle strade, Ghino deve essere considerato «infamis». Il carattere elogiativo del pezzo è evidente sin dall'inizio. Benvenuto si rivolge al lettore, come è sua abitudine, raccomandandogli di seguire una certa interpretazione del personaggio: questo non è affatto un personaggio da poco conto, è anzi «mirabile» e il suo ritratto merita gli elementi di un ritratto classico latino, sino all'aperta similitudine con i personaggi più leggendari della storia romana. Dapprima, gli elementi fisici, tutti implicanti un giudizio positivo; poi, tra gli attributi caratteriali, la prudentia e la largitas $^{16}$. Nella parte narrativa, invece, emergono i tratti più specifici del bandito cortese: egli non sopporta che le persone da lui catturate vengano uccise (quindi è moderato nell'uso della violenza), non ruba a chi è già povero, anzi, dà a chi è povero (in particolare agli studenti) denaro sufficiente per continuare il proprio percorso, concede ai mercanti di mantenere dei denari per i commerci, e infierisce soltanto sul «sacerdote ricco e grasso». Esattamente gli stessi elementi riscontrabili nelle ballate medievali che ci restano su Robin Hood, compreso l'aneddoto sui membri della Chiesa, ed evidenziati, in gran parte, nella ricostruzione di Hobsbawm.

Questa parte del commento dell'Imolese, che peraltro cita esplicitamente la novella del Decameron, sembra avere la struttura, a sua volta, di una novella di Boccaccio. Ritratto del personaggio, racconto dei suoi comportamenti più noti, con abbondanza di particolari aneddotici, uccisione del giudice (a commento del testo dantesco) e infine la morte, para-

16. Per quanto riguarda gli elementi fisici del ritratto, la connotazione niger pilo può sembrare una nota stonata: in realtà, c'è un richiamo alla descrizione di Azzolino da Romano in Inf., XII, 109 («E quella fronte ch'ha 'l pel così nero»). Là, però, la chiosa dell'Imolese si era arricchita di particolari: il pilus niger sarebbe in quel caso, secondo Benvenuto, un pelo sul naso di Azzolino che si drizzerebbe nei momenti di ira, e non l'intera sua chioma. Anche rispetto agli altri elementi del ritratto di Azzolino delineato dall'Imolese l'immagine di Ghino sembra in perfetto richiamo, per antitesi. Si dice infatti di Azzolino: «[...] vir pessimus, crudelissimus [...]. Exercuit magnas violentias et crudelitates, adeo quod quidam scripserunt ipsum fecisse mori quinquaginta milia hominum [...]. Scribunt aliqui, quod Ecerinus fuit corporis mediocris, niger, pilosus totus. Sed audio quod habebat unum pilum longum super nasum qui statim erigebatur, quando excandescebat in iram, et tunc omnes fugiebant a facie eius». 
gonata esplicitamente a quella di Cesare, in un crescendo di stile, sino ad arrivare ad uno stile alto, tragico. E proprio una tragedia, secondo Benvenuto, potrebbe essere scritta su Ghino. È questo un dato estremamente interessante, al di là di tutte le connotazioni che vengono date del personaggio. Una tragedia su Ghino, così come una tragedia è stata scritta su Ezzelino da Romano, peraltro citata con ammirazione dall'Imolese ${ }^{17}$ ? Certo, qui la valutazione sarebbe ribaltata. La storia di Ghino, però, potrebbe così "scalare" diversi generi letterari, sino al più alto.

A proposito del rapporto del bandito cortese con la Chiesa e con la devozione religiosa, si apre un campo che ci spinge a ulteriori osservazioni e indagini. Riguardo a Ghino, innanzitutto, occorre notare che se in questo brano egli si dimostra ostile, e in maniera sarcastica, nei confronti del rappresentante della religione, nella novella di Boccaccio, nonostante il racconto prenda avvio dal rapimento di un ecclesiastico notoriamente tra i più ricchi dell'epoca, oltre al finale atteggiamento magnanimo, si manifesta un atteggiamento di devozione diretta nei confronti di Dio stesso, che scavalca, in maniera ostentatamente provocatoria, l'autorità religiosa terrena. Dice infatti l'ambasciatore di Ghino all'abate: «Messere, voi siete in parte dove, dalla forza di Dio in fuori, di niente si teme per noi» ${ }^{18}$. Nell'insofferenza ad ogni genere d'autorità, la masnada di banditi alle dipendenze di Ghino non rinnega la «forza» divina e la riconosce come unica in grado di suscitare timore e obbedienza.

Ebbene, una forma di devozione religiosa slegata dal riconoscimento dell'autorità ecclesiastica si ritrova anche nelle ballate medievali di Robin Hood (la sua devozione, in particolare, è per Maria, e ciò lo porta addirittura a rischiare la vita per partecipare in città ad un rito solenne in suo onore); si ritrova anche, però, in altri personaggi di fuorilegge della tradizione letteraria italiana, che, pur non essendo affatto presentati come personaggi positivi, manifestano almeno uno dei tratti caratteristici del bandito cortese.

Un caso evidente è nella novella 39 del Novelliere di Sercambi. Il protagonista è un nobile (un conte, come si dice subito nell'incipit della novella) il quale assomma in sé ogni «mala condizione»:

17. Sempre nel commento ad Inf. XII, 109-110, Benvenuto afferma: «[...] vocatus Ecerinus, sicut scribit Mussattus Paduanus, Musarum amicus, in Tragoedia quam fecit de Eccerino in qua fingit quod fuit genitum a patre Diablo".

18. BoCCACCIO, Decameron, X, 2, 9. La ricchezza dell'abate di Cluny è esplicitamente menzionata ad inizio di novella («venne a corte l'abate di Clignì, il quale si crede essere un de' più ricchi prelati del mondo»). 
Fu un conte di quelli da Bruscola, del contado e giurisdizione di Bologna, il quale possedea alcune terre e fortezze innella montagna, nomato lo conte Sparaleone, omo di gran superbia e crudeltà e d'ogni mala condizione. E non stante che lui fusse malvagio e reo - ancora a' suoi famigli comandava che ogni male facessero -, e' pur non era però tanto malvagio, che almeno questo poco di bene facea: che ogni dì la mattina quando si levava, per lo dì dicea una avemaria, e la sera ne dicea per la notte un'altra. E questo era tutto lo bene che questo conte facea, né mai altro bene si disse che lui facesse. Avea questo conte molti maliscalzoni, e ladroncelli e d'ogni cattiva condizione, ai quali avea comandato che ogni dì facessero o furto o rubaria o micidio; e più, che a tutti, sotto grave pena ditto loro che mai persona che trovassero innel suo terreno che a lui per neuno modo si presentasse, ma che rubato che fusse quello uccidessero. E ogni cosa crudele li piacea più che le pietose. E per questo modo moltissimi prelati, mercadanti et altre buone persone, oltra le rubarie a loro fatte, erano stati morti ${ }^{19}$

In effetti, egli si comporta esattamente come un feroce anti-Ghino (per esempio, dopo averli derubati, uccide tutti coloro che hanno attraversato il suo territorio), ma c'è una circostanza che nobilita le sue azioni terribili e lo salva dalla dannazione eterna: egli ha una tale devozione religiosa che ogni giorno recita una preghiera mattutina e una preghiera serale. Quando il demonio, travestitosi da cuoco, cercherà di guadagnare la sua anima, un "angelo mandato da Dio», sotto le sembianze di un pellegrino, riuscirà a salvarlo proprio in virtù delle preghiere quotidiane del personaggio.

Il motivo, presente anche nella Legenda aurea e poi ripreso senza sostanziali varianti in tanti testi europei, si ritrova in Italia nel De castellano e nel De pirata di Bovensin de la Riva, all'interno delle Laudes de virgine Maria $^{20}$. Nel primo componimento, in particolare, esattamente come poi in Sercambi, il diavolo si traveste da cantiniere per cercare di conquistare l'anima del bandito, ma deve lottare con un angelo che, in virtù delle preghiere e della devozione a Dio del malvagio peccatore, gli impedisce di ottenere definitivamente quel trofeo.

Elementi del bandito cortese, dunque, anche là dove dell'audacia e della libertà dai vincoli sociali propri di quel personaggio non si intende in nessun modo dare una valutazione positiva. Nello stesso Novelliere di Sercambi, per esempio, sono diverse le novelle che hanno per protagonisti banditi o pirati, ma in esse sono messi in risalto la ferocia e la spietatezza,

19. Giovanni Sercambi, Il Novelliere, a cura di G. Sinicropi, Firenze, Le Lettere, 1995, vol. I, pp. 374-375.

20. BonVESIN DE LA RIVA, Laudes de Virgine Maria, in Le opere volgari di Bonvesin da la Riva, a cura di Gianfranco Contini, Roma, Società Filologica Romana, 1941, vol, I, pp. 215-218 e 218 221. 
e l'esito della loro vicenda è sempre la giusta punizione data dall'autorità statale o da singoli personaggi eroici. Nella maggior parte dei casi, poi, tale punizione viene ostentatamente descritta dal testo, con un rilievo particolare per gli aspetti più macabri (l'impiccagione, la pena del bruciare vivi, ecc.). La posizione dell'autore al riguardo è inequivocabile.

Nella novella 84, per esempio, è di nuovo protagonista un anti-Ghino: un «malandrino» che ha dimora nel castello di Montalto (sulla via dei pellegrini per Roma, come Ghino), tale Suffilello, deruba e fa uccidere tutti coloro che passano dal territorio che controlla, finché non rapisce la contessa d'Artois, con l'intenzione di gettarla da un dirupo dopo averla letteralmente spogliata di tutto. È lei, però, la protagonista e l'eroe positivo del racconto, e con un abile inganno riesce a gettare il brigante dallo stesso dirupo e a fare impiccare, vivi o morti che siano, tutti i componenti della masnada.

Nella novella 88 è di nuovo protagonista la stessa contessa, questa volta nei suoi territori francesi, impegnata a sterminare (bruciandoli vivi in un casolare oppure facendoli impiccare) i malandrini che operano lungo la strada per Parigi. Qui il narratore precisa che tra di essi vi sono anche uomini "gentili», ossia nobili datisi alla macchia. Neanche per loro, però, vi è una possibilità di salvezza o uno spiraglio di pietà, e nessuna loro caratteristica risulta quantomeno ambigua per la valutazione finale. Uguale esito impietoso e persino inquietante nelle novelle 87 e 82: la prima ha per protagonisti un gruppo di malandrini storpi, poi scoperti e impiccati; la seconda, sostanziale riscrittura della novella di Boccaccio di Rinaldo d'Esti (Decameron, II, 2), ha come riferimento la leggenda di San Giuliano, e fa concludere con l'impiccagione la vicenda dei briganti.

Sempre in Sercambi, però, compare un ulteriore elemento che vivacizza il nostro quadro. C’è un'altra figura di fuorilegge, oltre al conte da Bruscola, che ha un comportamento non univocamente condannato. Si tratta di un pirata che salva una delle vittime dei suoi rapimenti per la brillante risposta che riesce a dargli (novella 54). Il pirata è Piero da Rabat, "corsale crudelissimo» pronto ad uccidere annegandoli tutti gli uomini delle navi che ha depredato. Sen nonché uno di questi prigionieri, un francese, riesce a salvarsi la vita con un «bel motto" che ben dispone il pirata: vedendo l'acqua nella quale sta per essere gettato, dopo due giorni di digiuno, gli dice: «O sire, ciest est trou gran boire a si petit mangier». Dunque, un uomo "crudelissimo" inaspettatamente ravveduto di fronte all'intelligenza dell'interlocutore. $\grave{E}$ anch'esso una forma di fuorilegge cortese, che dimostra elementi di liberalità. 
Le figure dei pirati, fuorilegge che godono di uno statuto di libertà dai vincoli sociali ancora maggiore rispetto a quello dei banditi di strada, sono anch'esse numerose nella novellistica medievale, e nella maggior parte dei casi la loro caratterizzazione non differisce di molto da quella dei banditi di strada. In alcune circostanze, però, essi suscitano nei narratori simpatie più marcate.

Per ritornare al Decameron, da cui era partito il nostro excursus sui testi, sono diverse le novelle in cui compare questo tipo di personaggio, le cui fonti sono facilmente rintracciabili perlomeno nel romanzo antico latino e greco. Nella maggior parte dei casi si tratta di elementi di puro motore narrativo (il rapimento, l'abbandono, il ricongiungimento o l'agnizione, come nella novella II, 6 , che ha per protagonista Madama Beritola); ma in alcune circostanze i personaggi dei pirati diventano essi stessi protagonisti. È il caso per esempio di Landolfo Rufolo (II, 4), che diventa corsale (è questo il termine più usato da Boccaccio) per recuperare il patrimonio perduto (e si noti che la sua scelta e la sua azione non sono affatto connotate negativamente nel testo).

Ma il caso per noi più significativo è quello di Paganino da Mare (II, 10), pirata che rapisce la moglie di un giudice pisano, secondo il consolidato schema già proprio del romanzo antico. Qui il pirata è di gran lunga l'eroe positivo, colui che, seguendo uno stile di vita libero, riesce davvero a realizzare pienamente la propria umanità, e ad avere un comportamento ispirato a valori etici assoluti, privi di condizionamenti e impedimenti. Messer Riccardo di Chinzica, giudice pisano di grande ingegno ma di età avanzata e di poche doti "corporali», riesce ad ottenere in sposa la giovanissima Bartolomea, ma, seguendo rigorosamente i precetti di astinenza a suo dire dettati dalla Chiesa, non arriva a soddisfarne adeguatamente il desiderio sessuale. La donna, rapita un giorno da Paganino, scopre una vita radicalmente diversa e decide di non tornare più dal marito. Questi morirà di disperazione e la donna e il famoso pirata potranno regolarmente sposarsi. Paganino, nominato nella sintesi della novella con l'aggiunta del toponimo dove ha sede il suo covo (Monaco), ma nel resto della novella indicato con il nome di famiglia («da Mare», nobile famiglia genovese), ha fin da subito un comportamento libero e sicuro e, come il capo dei pirati di Eliodoro, è attratto dalla bellezza della giovane donna in maniera assoluta, tanto da non anteporle nulla:

E mentre che essi più attenti stavano a riguardare, subito una galeotta di Paganin da Mare, allora molto famoso corsale, sopravvenne e, vedute le barche si dirizzò a loro; le quali non poteron sì tosto fuggire, che Paganin non giugnesse quella ove eran le donne: nella quale veggendo la bella donna, senza altro volerne, quella veggente mes- 
ser Riccardo che già era in terra, sopra la sua galeotta posta andò via [...]. A Paganino, veggendola così bella, parve star bene; e non avendo moglie, si pensò di sempre tenersi costei, e lei che forte piangea cominciò dolcemente a confortare. E venuta la notte, essendo a lui il calendaro caduto da cintola e ogni festa o feria uscita di mente, la cominciò a confortar co' fatti, parendogli che poco fossero il dì giovate le parole; e per sì fatta maniera la racconsolò, che, prima che a Monaco giugnessero, e il giudice e le sue leggi le furono uscite di mente, e cominciò a viver più lietamente del mondo con Paganino; il quale, a Monaco menatala, oltre alle consolazioni che di dì e di notte le dava, onoratamente come sua moglie la tenea. (II, 10)

Specularmente, anche la donna si adatta immediatamente allo stile di vita del pirata e condivide infatti con lui la capacità di perseguire con fermezza ciò che ritiene giusto per sé. Quando il marito arriverà nel covo dei pirati per riprendersi la moglie, il contrasto tra i due personaggi maschili sarà nettissimo, e sarà la donna, invitata dal bandito a parlare «come le piacesse» a dimostrare la distanza tra i due e la sua scelta:

Imaginossi messer Riccardo che ella questo facesse per tema di Paganino, di non volere in sua presenza confessar di conoscerlo: per che dopo alquanto chiese di grazia a Paganino che in camera solo con essolei le potesse parlare. Paganin disse che gli piacea, sì veramente che egli non la dovesse contra suo piacere basciare; e alla donna comandò che con lui in camera andasse e udisse ciò che egli volesse dire e come le piacesse gli rispondesse. [...] «[...] E dicovi così, che qui mi pare esser moglie di Paganino e a Pisa mi pareva esser vostra bagascia, pensando che per punti di luna e per isquadri di geometria si conveniano tra voi e me congiugnere i pianeti, dove qui Paganino tutta la notte mi tiene in braccio e strignemi e mordemi, e come egli mi conci Dio vel dica per me [...]. (II, 10)

Paganino da Mare dimostra dunque cortesia nei confronti della donna (dicendole di parlare liberamente con il marito, mostra di darle piena dignità). È proprio questo suo comportamento a ridare alla donna il pieno possesso della propria persona, del proprio corpo ma anche della propria sfera decisionale, e dunque il personaggio del pirata ha nel racconto il ruolo di eroe positivo e nello stesso tempo maieutico nei confronti del personaggio femminile.

Riguardo ai pirati, come dicevo, alla libertà dai vincoli sociali che li rende più facilmente protagonisti positivi, si aggiunge la condizione di libertà fisica nello spazio, e in uno spazio come il mare, che sembra rendere effettivamente nulle le regole ordinarie delle comunità civili. Ancor più che $\mathrm{i}$ banditi di terra, i pirati si escludono totalmente e per periodi molto più prolungati dalla convivenza civile e dunque è ancora più agevole applicare su di loro una trasfigurazione letteraria. Non è difficile trovare altre storie in cui di un pirata venga data una connotazione positiva. Nel Novellino di Masuccio Salernitano, alla novella 22, per esempio, 
Nicolao d'Agnito, "gentilomo trapanese» "nei dì suoi famosissimo corsalo» nelle vicine coste «di Barbaria», abbandona la pirateria quando sposa una donna trapanese, ma è pronto a riprendere un «lignetto da corseggiare con i suoi cari compagni» quando la moglie scappa in Africa con un servo moro e lui intende vendicarsi. Il narratore considera positivamente la sua «animosità»e del tutto naturale l'attività della pirateria ${ }^{21}$.

Riguardo la pirateria nella narrativa, in contiguità, in effetti, con il mondo del banditismo, la letteratura antica è fonte non solo per quella funzione di motore e snodo della vicenda, ma anche per la tradizione di un personaggio che contribuisce a mio parere sia nella rappresentazione genericamente elogiativa dei pirati, come paladini di libertà e coraggio, che abbiamo visto, sia nella piena formazione del personaggio eroico del bandito cortese. Ne ritroviamo un'esplicita ripresa nell'Orlando innamorato, ma la fonte antica, probabilmente, ha agito già in precedenza nella strutturazione dei nostri personaggi. Quando Brandimarte si trova a duellare con il malandrino Barigaccio e gli riconosce un grandissimo valore nelle armi, lo invita ad abbandonare la vita da brigante e a diventare cavaliere, chiedendosi anzi come mai abbia fatto quella scelta. Così gli risponde Barigaccio:

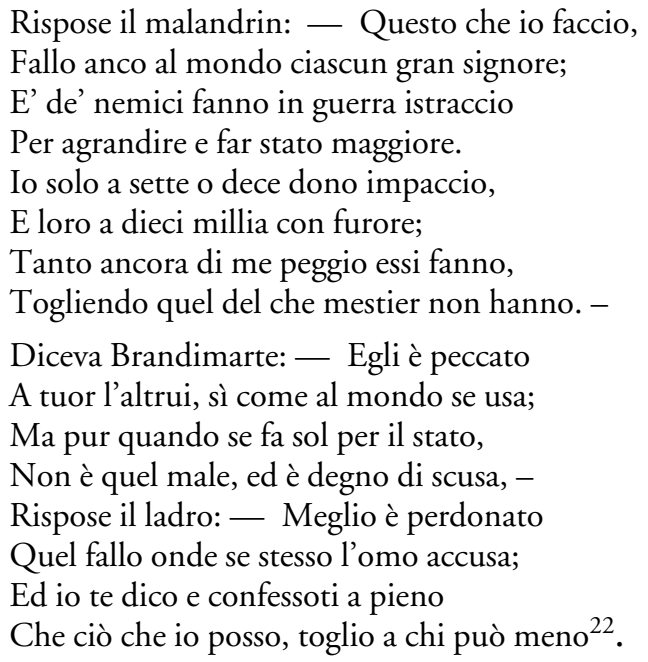

21. Masuccio Salernitano, Il Novellino, a cura di Giorgio Petrocchi, Firenze, Sansoni, 1991.

22. II, XIX, 40-41, in Matteo Maria BoIARdo, Opere, a cura di A. Tissoni Benvenuti, Milano, Ricciardi, 1999. 
Ciò che fa il «ladro» lo fa «al mondo ciascun gran signore» che voglia "agrandire e far stato maggiore», con la differenza che un signore danneggia diecimila persone, mentre un comune bandito sette o dieci. Il motivo, come è noto, è presente nel De civitate Dei (IV, 4) di Agostino, che a sua volta cita un perduto passo ciceroniano del De re publica, il quale riporta una tradizione diffusa nella produzione narrativa antica su Alessandro Magno $^{23}$. Soltanto, il malandrino di strada di Boiardo è in realtà un pirata nella tradizione che ha come interlocutore Alessandro, a testimoniare in effetti una facile sovrapposizione tra le due figure.

Nel brano di Agostino, dunque, un pirata, incontrato da Alessandro e da lui invitato a spiegare le ragioni della sua condizione, risponde eleganter et veraciter al re:

Eleganter enim et veraciter Alexandro illi Magno quidam comprehensus pirata respondit. Nam cum idem rex hominem interrogaret, quid ei videretur, ut mare haberet infestum, ille libera contumacia: «Quod tibi», inquit, «orbem terrarum; sed quia id ego exiguo navigio facio, latro vocor; quia tu magna classe, imperator».

Solo perché opera con una piccola imbarcazione, il pirata viene considerato "predone», mentre chi agisce allo stesso modo, ma con una grande flotta, sarà chiamato imperatore. La risposta intende dimostrare quanto gli stati costituiti siano simili ai latrocini là dove è assente la giustizia: il pirata, con l'eleganza e la libertà nel parlare, smaschera l'ipocrisia verbale e invita a non giudicare sulla base di apparenze e di categorie sociali effimere.

Con una funzione simile l'episodio viene citato di frequente tra Trecento e Quattrocento: nei Gesta Romanorum, per esempio, nel Dittamondo di Fazio degli Uberti (e in maniera più articolata nel relativo commento di Guglielmo Capello), nel Dialogus creaturarum $^{24}$. In tutti questi testi, però, la risposta del pirata ad Alessandro è più articolata, e la vicenda ha un seguito narrativo (che anche Boiardo avvia, ma poi decide

23. Il passo ciceroniano è noto, oltre che dalla citazione agostiniana, grazie al De compendiosa doctrina di Nonio Marcello (alla voce habere infestum e alla voce myoparone): «Nam cum quaereretur ex eo, quo scelere impulsus mare haberet infestum uno myoparone, "Eodem", inquit, "quo tu orbem terrae"” (CICERONE, De re publica III, 24). Sull'argomento, si veda EDOARDO FuMAGALLI, Appunti sul brigante Barigaccio nell'Orlando innamorato, in «Studi umanistici piceni», 19 (1999), pp. 88-93.

24. Gesta Romanorum, a cura di H. Oesterley, Hildesheim, Olms, 1963, $\$ 118$ (De Alexandro et Dionide pirata); FAZIO DEgLI UberTI, Il Dittamondo, IV, II, 70-72, in ID., Il Dittamondo e le rime, a cura di G. Corsi, Bari, Laterza, 1952; Dialogus creaturarum, 79 (De perdice fure), in Die beiden ältesten lateinischen Fabelbücher des Mittelalters: des Bischofs Cyrillus Speculum sapientiae und des Nicolaus Pergamenus Dialogus creaturarum, a cura di J.G.Th. Grasse, Hildesheim, Olms, 1965. 
di ribaltare): Alessandro, ammirato di fronte alle parole del pirata, decide di modificare la sua fortuna perché possa effettivamente essere migliore e lo rende «de latrone» "princeps»:

[...] «Et quare te orbis terrarum? Sed quia ego illud ago galea una, latro vocor; tu vero mundum opprimens navium multitudine magna diceris imperator. Sed si fortuna circa me mansuesceret, fierem melior; at contra, quo tu fortunatior, tanto eris deterior». Alexander respondit: «Fortunam tibi mutabo, ne malitia suae fortunae sed meritis asscribatur». Sicque ditatus est per eum, de latrone factus est princeps et mirabilis zelator iustitiae. ${ }^{25}$

La vicenda della "conversione" del bandito, resa possibile dalla sua abilità oratoria e allo stesso tempo dal gesto magnanimo di un potente, è del tutto analoga a quella di Ghino nella novella di Boccaccio, che non esiterei a mettere in relazione con questi testi: l'intervento del potente serve a neutralizzare uno svantaggio prodotto dalla fortuna, e l'abilità e l'ingegno oratorio del protagonista, che lo rendono eroe positivo, hanno la funzione di scatenare tale intervento. Il bandito diventa dunque una funzione narrativa che innesca la possibilità di rappresentare un riequilibrio tra differenze sociali considerate prive di un giusto fondamento. In fondo, ciò che sostiene Hobsbawm a proposito del banditismo sociale, e ciò che lasciano intravedere personaggi come il Ghino di Benvenuto da Imola o il Robin Hood delle ballate medievali inglesi.

La proposta che Brandimarte fa a Barigaccio, invece, cioè di abbandonare la vita da brigante per diventare onesto cavaliere, non ha lo stesso esito: Brandimarte, innanzitutto, non considera iniquo il rubare o l'uccidere "per il stato", mentre Barigaccio, da parte sua, ribadisce una sorta di legge del più forte, per cui continuerà a togliere «a chi può meno». Tra $\mathrm{i}$ due c'è dunque totale incomunicabilità, e Brandimarte, paladino nel racconto, avrà la meglio su di lui con le armi.

Il cerchio si chiude: se i banditi cortesi, eroi positivi a tutto tondo (Benvenuto da Imola) o eroi ambivalenti che lasciano supporre su di essi la circolazione di una leggenda ben più ampia e articolata di quanto i testi

25. Gesta Romanorum, cit., $\$ 118$. Di poco più articolato il Dialogus creaturarum: «[...] Si solus captus fuisset Alexander, latro esset, si ad nutum Dionidis populi famulentur, erit Dionides imperator; me fortunae iniquitas, te fastus intolerabilis et inexplicabilis avaritia furem facit. Si fortuna mutaretur, fierem forte melior, ac tu, quo fortunatior, eo nequior eris. Miratus Alexander de piratae constantia dixit: experiar, an futurus sis melior, fortunam ego tibi mutabo, ut non ei anmodo quae deliqueris, sed tuis potius moribus adscribatur. Et eum adscribi fecit militiae, ut posset exinde salvis legibus militare» (dialogo 79). Più sintetico, ma identico nei riferimenti il Dittamondo: «Seguia Dionides, del qual si sona / che 'l mar rubava e che parlò sì vivo, / che acquistò terra e scampò la persona» (IV, II, 70-72). 
non rappresentino (Boccaccio, Francesco da Barberino, probabilmente Dante), suscitano un interesse rilevante nella letteratura trecentesca e quattrocentesca, con Boiardo quell'interesse sembra spegnersi. Prevale la ragion di stato, la legalità assoluta, alla quale è subordinata qualsiasi altra azione. Bisognerà attendere I masnadieri di Schiller per ritrovare, pienamente realizzato, il personaggio del bandito gentiluomo. 